\title{
AN EXPONENTIAL TURNPIKE THEOREM FOR DISSIPATIVE DISCRETE TIME OPTIMAL CONTROL PROBLEMS
}

\author{
TOBIAS DAMM*, LARS GRÜNE ${ }^{\dagger}$, MARLEEN STIELER ${ }^{\ddagger}$, AND KARL WORTHMANN $^{\S}$
}

\begin{abstract}
We investigate the exponential turnpike property for finite horizon undiscounted discrete time optimal control problems without any terminal constraints. Considering a class of strictly dissipative systems we derive a boundedness condition for an auxiliary optimal value function which implies the exponential turnpike property. Two theorems illustrate how this boundedness condition can be concluded from structural properties like controllability and stabilizability of the control system under consideration.
\end{abstract}

Key words. turnpike property, optimal control, dissipativity, stabilizability, controllability, model predictive control

AMS subject classifications. 49K30, 49K21, 93B05

1. Introduction. An optimal trajectory of a control problem is said to have the turnpike property if it first approaches an equilibrium state, stays close to it for a while and finally turns away from it again. The name turnpike property is motivated by the analogy of the behavior of the optimal trajectories to the strategy of driving from a point $A$ to $B$ on a road system consisting of highways ("turnpikes") and smaller roads. When the distance from $A$ to $B$ is sufficiently long, it is typically time optimal to first drive from $A$ to the nearest highway ( $\hat{=}$ move to the equilibrium), drive on the highway towards the nearest exit to $B$ (㐱 stay near the equilibrium) and then exit in order to reach $B$ via smaller roads ( $\hat{=}$ turn away from the equilibrium).

The turnpike property has been studied at least since the work of von Neumann in 1945 [22] and Dorfman, Samuelson and Solow in 1958 [11, p. 331]. Since then it has been observed in many optimal control problems. There is a vast amount of literature on sufficient conditions for this phenomenon to hold, see, e.g., [9, Section 4.4] or [28], particularly in economics, see, e.g., [21] and the references therein. However, only very few references treat the case of exponential turnpike which we consider in this paper for nonlinear undiscounted discrete time optimal control problems without terminal constraints. Our main motivation for studying this property is its recently discovered importance for obtaining convergence results in economic model predictive control (MPC) without terminal constraints, see [13]. The particular interest in exponentially fast versions of the turnpike property is triggered by the fact that, compared to slower turnpike properties, the exponential turnpike property allows to conclude additional qualitative properties of the MPC closed loop solution, like trajectory convergence and approximate finite time optimal transient behavior, for details see Section 3, below. While some exponential turnpike theorems can be found in the literature, our approach extends these results in various ways, e.g., by assuming only strict

\footnotetext{
*Mathematical Institute, Technical University of Kaiserslautern, 67653 Kaiserslautern, Germany, damm@mathematik. uni-kl.de

${ }^{\dagger}$ Mathematical Institute, University of Bayreuth, 95440 Bayreuth, Germany, lars.gruene Quni-bayreuth.de, supported by the European Union under the 7th Framework Programme FP7PEOPLE-2010-ITN, Grant agreement number 264735-SADCO

${ }_{\ddagger}$ Mathematical Institute, University of Bayreuth, 95440 Bayreuth, Germany, marleen.stieler Quni-bayreuth.de, supported by the International Doctorate Program "Identification, Optimization and Control with Applications in Modern Technologies" within the Elite Network of Bavaria

$\S$ Institute for Mathematics, Technical University of Ilmenau, 98693 Ilmenau, Germany, karl worthmann@tu-ilmenau.de, supported by the DFG Priority Program 1305, Grant Gr1569/11-2
} 
dissipativity instead of strict convexity. A detailed comparison is provided in Section 3 , below. We emphasize that although some of the ingredients of our analysis are well known in the turnpike literature - like dissipativity which was used before, e.g., in [8] - establishing exponential turnpike requires significantly different proof techniques than proving conventional turnpike.

The particular dissipativity property we use here is motivated by recent results for economic MPC with terminal constraints [2, 4, 5, 10], where dissipativity turns out to play a crucial role for stability considerations. Besides merely imposing this property, we also provide sufficient conditions for strict dissipativity of affine linear quadratic problems. For strictly dissipative systems, we first give a condition on a value function of an auxiliary problem, bearing some similarity with conditions from $[16,12,15]$ for the analysis of stabilizing MPC schemes. Under this condition we can then conclude the exponential turnpike property in Theorem 5.6. Since the assumptions of this theorem are quite implicit, in Theorems 6.2 and 6.5 we show for two different classes of systems how these assumptions can be verified using structural conditions like stabilizability and controllability.

The paper is organized as follows. After defining our notation, in Section 2 we explain our setting and problem formulation. In Section 3 the role of the exponential turnpike property in model predictive control and the relation of our result to other exponential turnpike properties in the literature are discussed. In Section 4 we introduce the dissipativity property we need for our approach and investigate it for affine linear quadratic problems. In Section 5 we derive a sufficient condition in terms of a bound on an auxiliary optimal value function. Then, in Section 6 two examples of how this condition can be checked in terms of stabilizability and controllability assumptions on the underlying control system are presented. Section 7 concludes the paper. An auxiliary result on positive definite matrices is proved in the Appendix in Section 8.

Notation: With $\mathbb{N}, \mathbb{Z}, \mathbb{R}$ and $\mathbb{C}$ we denote the natural, integer, real and complex numbers, respectively. We write $\mathbb{N}_{0}:=\mathbb{N} \cup\{0\}$ and $\mathbb{R}_{0}^{+}:=\{r \in \mathbb{R} \mid r \geq 0\}$. With $\mathbb{C}_{>1}$, $\mathbb{C}_{<1}$ and $\mathbb{C}_{=1}$ we denote the exterior, the interior and the boundary of the complex unit disk. For $r \in \mathbb{R}$ we define $\lfloor r\rfloor:=\max \{k \in \mathbb{Z} \mid k \leq r\}$ to be the largest integer $\leq r$.

For a metric space $X$ with metric $d(\cdot, \cdot)$ and some point $x^{e} \in X$, we write the distance of $x \in X$ to $x^{e}$ briefly as $|x|_{x^{e}}:=d\left(x, x^{e}\right)$. A set $M \subset X$ is called bounded if there exists $C>0$ with $d(x, y) \leq C$ for all $x, y \in M$. A function $g: \mathbb{X} \rightarrow \mathbb{R}$ with $\mathbb{X} \subset X$ is called radially unbounded on $\mathbb{X}$ if for each $C>0$ there exists a bounded set $M \subset X$ such that $\inf _{x \in \mathbb{X} \backslash M} g(x) \geq C$, using the convention $\inf _{x \in \emptyset} g(x)=\infty$. The open ball with radius $\varepsilon>0$ around $x \in X$ is denoted by $B_{\varepsilon}(x):=\{y \in X \mid d(y, x)<\varepsilon\}$.

For a quadratic matrix $Q \in \mathbb{R}^{n \times n}$ we write $Q>0$ if it is positive definite and $Q \geq 0$ if it is positive semidefinite. The spectrum of a matrix $A \in \mathbb{R}^{n \times n}$ is denoted by $\sigma(A) \subset \mathbb{C}$.

Furthermore, we define the classes of comparison functions

$$
\begin{aligned}
\mathcal{L}_{\mathbb{N}} & :=\left\{\delta: \mathbb{N} \rightarrow \mathbb{R}_{0}^{+} \mid \delta \text { decreasing with } \lim _{k \rightarrow \infty} \delta(k)=0\right\}, \\
\mathcal{K} & :=\left\{\alpha: \mathbb{R}_{0}^{+} \rightarrow \mathbb{R}_{0}^{+} \mid \alpha \text { continuous, strictly increasing, and } \alpha(0)=0\right\}, \\
\mathcal{K}_{\infty} & :=\{\alpha \in \mathcal{K} \mid \alpha \text { is unbounded }\} .
\end{aligned}
$$


2. Problem formulation. We consider nonlinear discrete time control systems given by

$$
x(k+1)=f(x(k), u(k))
$$

with state space $X$ and set of control values $U$, where $X$ and $U$ are metric spaces. State and control constraints are modelled by a set $\mathbb{Y} \subseteq X \times U$ which is decomposed into the sets

$$
\mathbb{X}:=\{x \in X \mid \text { there exists } u \in U \text { with }(x, u) \in \mathbb{Y}\}
$$

and, for each $x \in \mathbb{X}$

$$
\mathbb{U}(x):=\{u \in U \mid(x, u) \in \mathbb{Y}\} .
$$

For some results we will assume that the set $\mathbb{Y}$ is of the form

$$
\mathbb{Y}:=\{(x, u) \in X \times U \mid g(x, u) \leq 0\}
$$

for a function $g: X \times U \rightarrow \mathbb{R}^{n}$, where the " $\leq$ " is to be understood componentwise. With $x_{u}(k, x), k=0, \ldots, N$, we denote the state trajectory emanating from initial state $x$ and manipulated by the sequence of input values $u=(u(0), \ldots, u(N-1)) \in$ $U^{N}$. Such a sequence $u$ is said to be admissible for state $x \in \mathbb{X}$ and $N \in \mathbb{N}$ if the conditions

$$
\left(x_{u}(k, x), u(k)\right) \in \mathbb{Y} \text { for all } k=0, \ldots, N-1 \quad \text { and } \quad x_{u}(N, x) \in \mathbb{X}
$$

hold. The set of all such sequences is denoted by $u \in \mathcal{U}^{N}(x)$.

For stage costs $\ell: X \times U \rightarrow \mathbb{R}$ and $N \in \mathbb{N}$ we consider the optimal control problem

$$
\text { minimize } J_{N}(x, u):=\sum_{k=0}^{N-1} \ell\left(x_{u}(k, x), u(k)\right) \quad \text { subject to } \quad u \in \mathcal{U}^{N}(x) .
$$

The corresponding optimal value function is given by

$$
V_{N}(x):=\inf _{u \in \mathcal{U}^{N}(x)} J_{N}(x, u)
$$

with the convention $V_{N}(x)=\infty$ if $\mathcal{U}^{N}(x)=\emptyset$. We assume that for each $N \in \mathbb{N}$ and $x \in \mathbb{X}$ with $\mathcal{U}^{N}(x) \neq \emptyset$ a minimizing control sequence in (2.3) exists, i.e., there is a control $u_{x, N}^{\star} \in \mathcal{U}^{N}(x)$ with

$$
J_{N}\left(x, u_{x, N}^{\star}\right)=\sum_{k=0}^{N-1} \ell\left(x_{u_{x, N}^{\star}}(k, x), u_{x, N}^{\star}(k)\right)=V_{N}(x),
$$

cf. [14, Section 3.4] for a discussion on this assumption.

In this paper, we are interested in the dynamical behavior of the optimal trajectories $x_{u_{x, N}^{\star}}$. To this end, we first define the notion of an equilibrium or steady state.

Definition 2.1. A point $x^{e} \in \mathbb{X}$ is called an equilibrium (or steady state) of (2.1) if there exists $u^{e} \in \mathbb{U}\left(x^{e}\right)$ with $f\left(x^{e}, u^{e}\right)=x^{e}$. In this case, the pair $\left(x^{e}, u^{e}\right) \in \mathbb{Y}$ is called an equilibrium pair. 
Given an equilibrium $x^{e}$, we are now interested in the question whether the optimal trajectories (2.5) stay in a neighborhood of $x^{e}$ for a certain time, where the diameter of this neighborhood shrinks to 0 as $N \rightarrow \infty$. This phenomenon is known as the turnpike property and its precise description is as follows.

DEFINITION 2.2. (i) We say that the optimal control problem has the turnpike property on a set $\mathbb{X}_{0} \subseteq \mathbb{X}$ if there exists an equilibrium $x^{e} \in \mathbb{X}$ such that the following condition holds.

For each $P \in \mathbb{N}$ there exists a function $\sigma_{P} \in \mathcal{L}_{\mathbb{N}}$ such that for any optimal trajectory $x_{u_{x, N}^{\star}}(\cdot, x)$ with $x \in \mathbb{X}_{0}$ and $N \geq P$ there is a set $\mathcal{Q} \subseteq\{0, \ldots, N\}$ with $\# \mathcal{Q} \geq P$ such that the inequality

$$
\left|x_{u_{x, N}^{\star}}(k, x)\right|_{x^{e}} \leq \sigma_{P}(N)
$$

holds for all $k \in \mathcal{Q}$.

(ii) The turnpike property from (i) is called exponential if $\sigma_{P}$ can be chosen as $\sigma_{P}(N):=C_{P} \theta^{N}$ for constants $C_{P}>0$ and $\theta \in(0,1)$.

The turnpike property can be observed for many optimal control problems and we illustrate it by two examples taken from [13]. The optimal trajectories were computed numerically by performing one prediction step with the MATLAB routine nmpc.m (cf. [14, Appendix A] and www.nmpc-book.com) which uses the fmincon optimization routine.

EXAMPLE 2.3. Consider the control system $x(k+1)=2 x(k)+u(k)$ with $X=U=$ $\mathbb{R}$, stage cost $\ell(x, u)=u^{2}$ and constraints $\mathbb{X}=[-0.5,0.5]$ and $\mathbb{U}=[-2,2]$. Figure 2.1 (left) shows the optimal trajectories for initial value $x=0.5$ and $N=5,10, \ldots, 40$. One sees that the trajectories approach the point $x^{e}=0$ closer and closer and stay inside a small neighborhood of this point for more and more time steps.
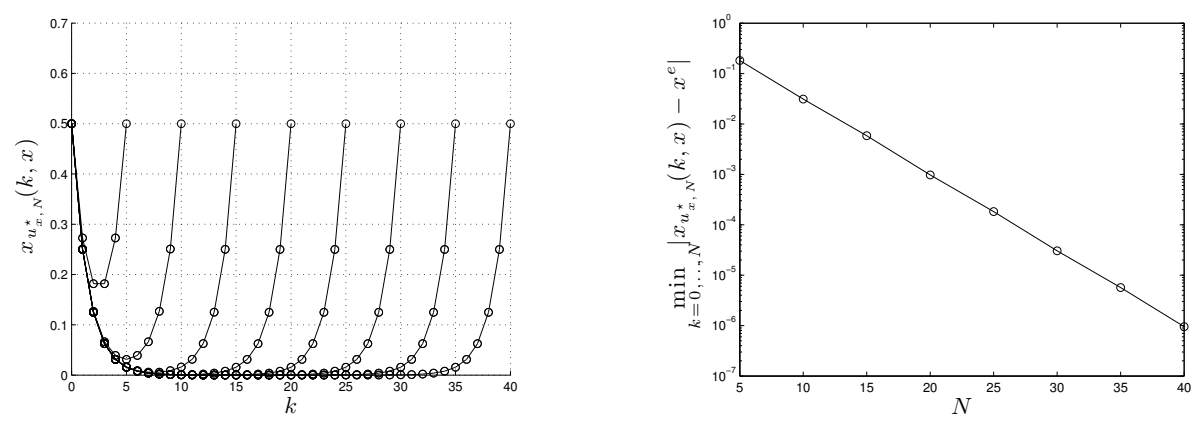

FIG. 2.1. Optimal trajectories $x_{u_{x, N}^{\star}}(k, x)$ for Example 2.3 for various $N$ (left) and minimal distance $\min _{k=0, \ldots, N}\left\|x_{u_{x, N}^{\star}}(k, x)-x^{e}\right\|$ to $x^{e}=0$ (right).

Figure 2.1 (right) shows the minimal distance of the optimal trajectories for various $N$ on a logarithmic scale. One sees that the distance decreases exponentially with increasing $N$.

EXAMPLE 2.4. The second example is a linearized continuously stirred tank reactor model with two dimensional affine linear dynamics

$$
x(k+1)=\left(\begin{array}{ll}
0.8353 & 0 \\
0.1065 & 0.9418
\end{array}\right) x(k)+\left(\begin{array}{r}
0.00457 \\
-0.00457
\end{array}\right) u(k)+\left(\begin{array}{l}
0.5559 \\
0.5033
\end{array}\right)
$$


with $X=\mathbb{R}^{2}, U=\mathbb{R}$ and stage cost $\ell(x, u)=\|x\|^{2}+0.05 u^{2}$. We use the state and control constraints $\mathbb{X}=[-100,100]^{2}$ and $\mathbb{U}=[-10,10]$.

Figure 2.2 shows the two components of the optimal trajectories for initial value $x=(4,15)^{T}$ and $N=10,20, \ldots, 100$. Here, one observes a turnpike property for the point $x^{e} \approx(3.546,14.653)^{T}$ indicated by the solid lines in the figures (we will see later how this point was computed).
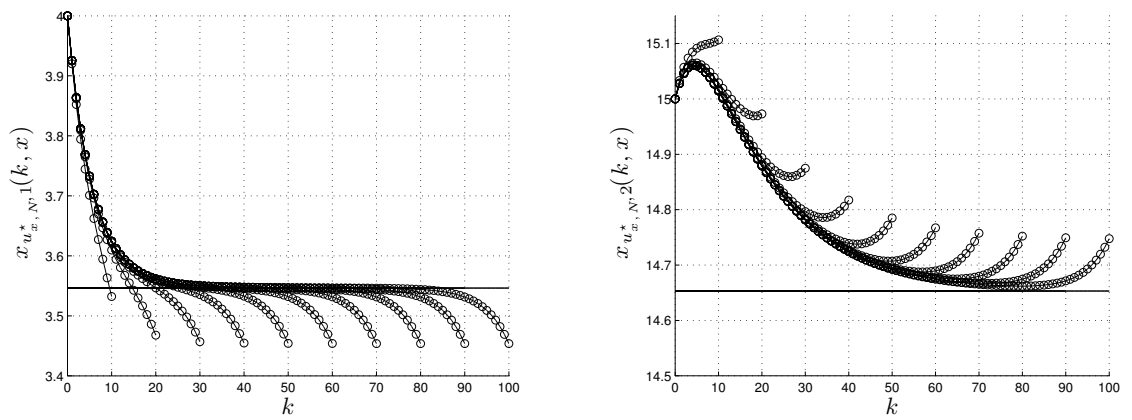

FIG. 2.2. Optimal trajectories $x_{u_{x, N}^{\star}}(k, x)$ for Example 2.4 for various $N, x_{1}$-component (left) and $x_{2}$-component (right)

Again, the minimal distance of the numerically computed optimal trajectories decreases exponentially in $N$ as shown in Figure 2.3.

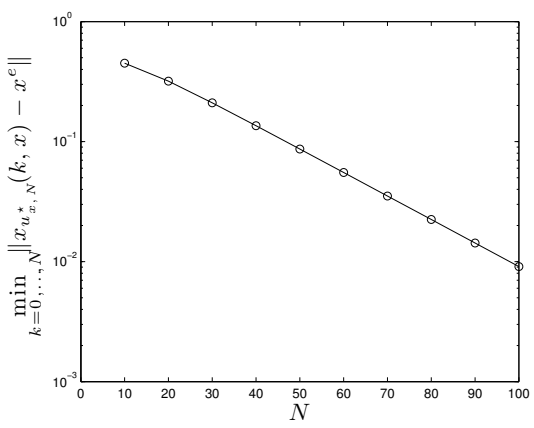

FIG. 2.3. Minimal distance $\min _{k=0, \ldots, N}\left\|x_{u_{x, N}^{\star}}(k, x)-x^{e}\right\|$ to $x^{e} \approx(3.546,14.653)^{T}$

3. Motivation. There are various reasons why turnpike properties are an interesting subject to study. First, the fact that this property is frequently observed for optimal trajectories naturally leads to the question of giving sufficient conditions on $f$ and $\ell$ under which this behavior can be rigorously proved. Results in this direction (for continuous time systems) can be found, e.g., in [9, Section 4.4]. Second, in many applications one is interested in the dynamical properties of optimal trajectories in order to understand the future behavior of optimally controlled systems, see, e.g., [21] and the references therein for economic applications.

Our main motivation stems from the recently observed importance of the turnpike property in model predictive control (MPC). In MPC, a so called MPC closed loop trajectory $x_{c l}(\cdot)$ is synthesized from the iterative solution of $(2.3)$ in the following way. 
0 . Fix some $N \in \mathbb{N}$, set $x_{c l}(0):=x_{0}$ and $k:=0$

1. Solve (2.3) for initial value $x=x_{c l}(k)$ and set $\mu_{N}\left(x_{c l}(k)\right):=u_{x, N}^{\star}(0)$

2. Set $x_{c l}(k+1):=f\left(x, \mu_{N}(x)\right), k:=k+1$ and go to 1 .

Defining the infinite horizon averaged performance of the resulting (infinitely long) trajectory by

$$
J_{\infty}^{c l}\left(x_{0}, \mu_{N}\right):=\limsup _{K \rightarrow \infty} \frac{1}{K} \sum_{k=0}^{K-1} \ell\left(x_{c l}(k), \mu_{N}\left(x_{c l}(k)\right)\right)
$$

the natural quantity to investigate is the difference of this value from the optimal infinite horizon averaged performance of the system.

In a series of papers $[2,4,5,10]$ it was shown - among other results - that if the system exhibits an optimal equilibrium $x^{e}$ (which we will define precisely in the next section) and if appropriate terminal constraints involving $x^{e}$ are imposed when minimizing $J_{N}\left(x_{c l}(k), u\right)$ in Step 1, then optimal performance of the MPC closed loop trajectories follows. Moreover, under a dissipativity condition (which will also be formally introduced in the next section) asymptotic stability of the optimal equilbrium for the closed loop system can be shown.

For the MPC algorithm in its "plain" form, i.e., without adding terminal constraints in Step 1, optimality of the MPC closed loop trajectory was investigated in [13]. For this setting, it turns out that the turnpike property is one of the decisive ingredients in order to conclude approximate optimality of the closed loop, where the gap to optimality tends to 0 as $N \rightarrow \infty$. If, in addition, the turnpike property is exponential, then convergence of the closed loop to a neighborhood of the optimal equilibrium $x^{e}$ can be shown and the closed loop trajectory will not only be approximately optimal in the infinite horizon averaged sense but also on the finite horizon during its transient phase. More precisely, there exists a time $K \in \mathbb{N}$ such that among all trajectories starting in $x_{0}$ and reaching a neighborhood of $x^{e}$ until this time $K$, up to an error term vanishing as $N \rightarrow \infty$ the MPC closed loop trajectory will be the one with the lowest cost $J_{K}$. This "transient optimality" result is considerably stronger than infinite horizon averaged optimality, since infinite horizon averaged optimal trajectories can show arbitrarily poor performance on any finite time interval. Hence, in the context of MPC the exponential turnpike property does not only yield a quantitative improvement of the results but in fact yields qualitatively new results, which is one of our main motivations to study this property.

At a first glance, the exponential turnpike property appears to be a very strong condition. However, we were able to observe it numerically in many examples, including those presented in the preceding section. Hence, it should be possible to determine structural assumptions for this property to hold and this is what this paper is devoted to.

While the vast majority of turnpike theorems in the literature does not yield exponential decay, there are some exceptions in the economic literature. Exponential turnpike theorems for discounted problems are presented, e.g., in [21, Theorem 10.1] and $[6 \text {, Theorem (4.5) }]^{1}$. The technical assumptions in these references are, in general, difficult to compare to our setting since the dynamics are defined implicitly via a set $D \subset X \times X$ which in our setting would be $D=\{(x, f(x, u)) \mid(x, u) \in \mathbb{Y}\}$. However, major differences to our setting are that the discount factor needs to satisfy

\footnotetext{
${ }^{1}$ Although [6] was published earlier than [21] it contains a detailed comparison with [21, Theorem 10.1] in Section 6.
} 
$\rho<1$ which excludes the case $\rho=1$ treated here and that certain strict concavity assumptions (which translate to strict convexity for our minimization problem) are imposed on the stage cost, for details see the discussion in [6, Section 6]. Here, we do not necessarily assume strict convexity of $\ell$ and we note that this property fails to hold in Example 2.3, since $\ell(x, u)=u^{2}$ is only strictly convex in $u$ but not in $x$. Instead, we use a dissipativity condition which can also be satisfied if $\ell$ is not strictly convex, cf. Proposition 4.5. Dissipativity, see [8, 9], and related properties, see, e.g, [19, Condition 2.2], are already well known for establishing non-exponential turnpike for continuous time problems without assuming convexity or concavity. For finite and infinite dimensional linear quadratic continuous time problems, the recent paper [23] establishes exponential turnpike theorems via the use of Riccati equations (for an earlier Riccati approach to turnpike-like results see also [3]). While this approach yields similar results to ours in the linear quadratic case, our approach in this paper applies to general nonlinear nonquadratic problems and entirely avoids the use of Riccati equations. Stochastic versions of exponential turnpike theorems are presented in [1, Theorem 2] for finite and infinite horizon problems and in [20, Theorem 2] for infinite horizon problems. While the infinite horizon setting in these references differs considerably from our finite horizon setting, the finite horizon result in [1] bears some similarities to our result. However, among the various assumptions in [1], one of the major differences to our assumptions is the terminal constraint condition (6) of the form $x_{N}=0$ which excludes the application to model predictive control without terminal constraints. Indeed, the fact that we do not need to impose any terminal constraints can be seen as one of the main contributions of our result.

4. Dissipativity. Dissipativity has been recognized as an important systems theoretic property since the seminal papers by Willems [26, 27]. The (discrete time) version of the dissipativity property we employ in this paper is motivated by $[2,5,10]$ and uses the function $\ell$ as what is called the supply rate in general dissipativity theory.

DeFINITION 4.1. (i) Let $\left(x^{e}, u^{e}\right) \in \mathbb{Y}$ be an equilibrium pair of (2.1). We say that the optimal control problem (2.3) is dissipative if there exist functions $\lambda: \mathbb{X} \rightarrow \mathbb{R}$ and $\alpha: \mathbb{R}_{0}^{+} \rightarrow \mathbb{R}_{0}^{+}$such that the inequality

$$
\tilde{\ell}(x, u):=\ell(x, u)+\lambda(x)-\lambda(f(x, u)) \geq \alpha\left(|x|_{x^{e}}\right)+\ell\left(x^{e}, u^{e}\right)
$$

holds for all $(x, u) \in \mathbb{Y}$ with $f(x, u) \in \mathbb{X}$.

(ii) We call the problem strictly dissipative if it is dissipative with $\alpha \in \mathcal{K}_{\infty}$.

(iii) The function $\tilde{\ell}$ defined in (4.1) is called modified (or rotated) stage cost.

REMARK 4.2. Note that whenever necessary without loss of generality we can assume $\ell\left(x^{e}, u^{e}\right)=0$ and $\lambda\left(x^{e}\right)=0$ (and thus also $\tilde{\ell}\left(x^{e}, u^{e}\right)=0$ ) since adding constants to $\lambda$ and $\ell$ does neither change the optimal trajectories nor the validity of (4.1).

In order to better understand the dissipativity property from Definition 4.1 and in order to be able to verify it for certain classes of examples, in the remainder of this section we investigate this property (in its strict form) for finite dimensional affine linear systems

$$
x(k+1)=A x(k)+B u(k)+c
$$

with $X=\mathbb{R}^{n}, U=\mathbb{R}^{m}, A \in \mathbb{R}^{n \times n}, B \in \mathbb{R}^{n \times m}, c \in \mathbb{R}^{n}$ and constraint set $\mathbb{Y}$ of the form (2.2). We investigate both strictly convex stage costs $\ell$ as well as costs of the linear-quadratic form

$$
\ell(x, u):=x^{T} R x+u^{T} Q u+s^{T} x+v^{T} u
$$


for symmetric matrices $R \in \mathbb{R}^{n \times n}, Q \in \mathbb{R}^{m \times m}$ and vectors $s \in \mathbb{R}^{n}, v \in \mathbb{R}^{m}$, with $R \geq 0$ and $Q>0$, which are not strictly convex in case $R \ngtr 0$.

The key for verifying strict dissipativity for this class of systems relies on the observation that the equilibrium pair $\left(x^{e}, u^{e}\right)$ in Definition 4.1 satisfies $\ell\left(x^{e}, u^{e}\right) \leq$ $\ell\left(\tilde{x}^{e}, \tilde{u}^{e}\right)$ for all other equilibrium pairs $\left(\tilde{x}^{e}, \tilde{u}^{e}\right) \in \mathbb{Y}$ of $(2.1)$, with strict inequality in case of strict dissipativity. Hence, $\left(x^{e}, u^{e}\right)$ is the optimal equilibrium pair of the system.

For (4.2) and $\mathbb{Y}$ from (2.2), the optimal equilibrium pair in $\mathbb{Y}$ can be expressed as the solution to the following optimization problem

$$
\begin{aligned}
& \min _{x \in X, u \in U} \ell(x, u) \\
& \text { s.t. } \quad x-A x-B u-c=0, \quad g(x, u) \leq 0 .
\end{aligned}
$$

Assuming that $g: X \times U \rightarrow \mathbb{R}^{p}$ is convex in $(x, u)$ and $\ell: X \times U \rightarrow \mathbb{R}$ is strictly convex in $(x, u)$ we can prove the following proposition, whose result is already mentioned (though not proved) in [10]. Here, for convenience of the reader, we provide a full proof.

Proposition 4.3. Consider the optimal control problem (2.3) with dynamics (4.2), strictly convex $\ell$ and constraint set $\mathbb{Y}$ defined via (2.2) with a convex function g. Assume that (4.4) has a global minimum $\left(x^{e}, u^{e}\right)$ and satisfies the following Slater condition: There exists a pair $(\hat{x}, \hat{u}) \in X \times U$ with

$$
\begin{aligned}
g(\hat{x}, \hat{u}) & <0, \\
\hat{x}-A \hat{x}-B \hat{u}-c & =0 .
\end{aligned}
$$

Then, there exists a vector $\nu \in \mathbb{R}^{n}$ such that the optimal control problem is strictly dissipative with $\lambda(x)=\nu^{T} x$. Moreover, if $\ell$ is of the form (4.3) and strictly convex, then $\alpha$ in (4.1) can be chosen as $\alpha(r)=C r^{2}$ for some $C>0$.

Proof. Due to the convexity assumptions on $\ell$ and $g$, the global minimum $\left(x^{e}, u^{e}\right)$ of (4.4) is unique. Since the Slater condition (4.5) is satisfied, [7, Section 5.9.1] implies the existence of multipliers $\left(\mu^{T} \nu^{T}\right)^{T} \in \mathbb{R}^{p+n} \backslash\{0\}$ satisfying

$$
\mu_{i} \geq 0 \quad \text { and } \quad \mu_{i}=0 \text { if } g_{i}\left(x^{e}, u^{e}\right)<0
$$

for each $i \in\{1,2, \ldots, p\}$ such that for all $(x, u) \in X \times U \backslash\left\{\left(x^{e}, u^{e}\right)\right\}$

$$
\ell\left(x^{e}, u^{e}\right)+\left(\begin{array}{l}
\mu \\
\nu
\end{array}\right)^{T}\left(\begin{array}{c}
g\left(x^{e}, u^{e}\right) \\
x^{e}-A x^{e}-B u^{e}-c
\end{array}\right)<\ell(x, u)+\left(\begin{array}{c}
\mu \\
\nu
\end{array}\right)^{T}\left(\begin{array}{c}
g(x, u) \\
x-A x-B u-c
\end{array}\right)
$$

where the strict inequality follows from the strict convexity of $\ell$.

We now define a function $L_{g}: X \times U \rightarrow \mathbb{R}$ by

$$
L_{g}(x, u):=\ell(x, u)-\ell\left(x^{e}, u^{e}\right)+\left(\mu^{T}, \nu^{T}\right)\left(\begin{array}{c}
g(x, u) \\
x-A x-B u-c
\end{array}\right) .
$$

From (4.7) we get

$$
L_{g}(x, u)>\left(\mu^{T}, \nu^{T}\right)\left(\begin{array}{c}
g\left(x^{e}, u^{e}\right) \\
x^{e}-A x^{e}-B u^{e}-c
\end{array}\right)=0 \quad \forall(x, u) \neq\left(x^{e}, u^{e}\right) .
$$


Additionally, (4.6) ensures $\mu^{T} g(x, u) \leq 0$ and hence

$$
L(x, u):=\ell(x, u)-\ell\left(x^{e}, u^{e}\right)+\nu^{T}(x-A x-B u-c) \geq L_{g}(x, u)
$$

for all $(x, u) \in \mathbb{Y}$.

Since $L(x, u)$ corresponds to our modified cost $\tilde{\ell}(x, u)$ if we set $\tilde{\ell}\left(x^{e}, u^{e}\right)=0$ and $\lambda(x)=\nu^{T} x$ showing

$$
L(x, u) \geq \gamma\left(\left\|\left(x-x^{e}, u-u^{e}\right)\right\|\right) \geq \gamma\left(\left\|x-x^{e}\right\|\right) \quad \forall(x, u) \in \mathbb{Y}
$$

for some class $\mathcal{K}_{\infty}$-function $\gamma: \mathbb{R}_{>0} \rightarrow \mathbb{R}_{>0}$ implies the assertion.

As a sum of convex functions $L: X \times U \rightarrow \mathbb{R}$ is convex and, as a consequence, continuous in the relative interior of its domain [24, Theorem 10.1] and, thus, on $X \times U$ [24, Corollary 10.1.1]. Since $L$ is, in addition, positive definite according to (4.9) and (4.8), [18, Lemma 4.3] can be used in order to conclude (4.10) if $L$ is radially unbounded $(L(x, u) \rightarrow \infty$ for $\|(x, u)\| \rightarrow \infty)$.

To this end, let us first assume that $\mathbb{Y}$ is unbounded. Let $r>0$ be arbitrarily given but fixed and $(x, u) \in \mathbb{Y}$ be given such that $\|(x, u)\|>r$ holds. Since a continuous function attains its minimum on a given compact set, (4.9) and (4.8) imply that there exists a real constant $c>0$ such that

$$
L(x, u) \geq c \quad \forall(x, u) \in \partial B_{r}\left(x^{e}, u^{e}\right) \cap \mathbb{Y}
$$

holds where $\partial B_{r}\left(x^{e}, u^{e}\right)$ is defined as $\left\{(x, u) \in X \times U:\left\|\left(x-x^{e}, u-u^{e}\right)\right\|=r\right\}$. Now, consider the straight line segment that joins $(x, u)$ and $\left(x^{e}, u^{e}\right)$. Due to convexity of $\mathbb{Y}$, there is a $(\bar{x}, \bar{u}) \in \partial B_{r}\left(x^{e}, u^{e}\right) \cap \mathbb{Y}$ lying on the line segement, i.e. there is a $\eta \in(0,1)$ such that $(\bar{x}, \bar{u})=\eta(x, u)+(1-\eta)\left(x^{e}, u^{e}\right)$ with $\eta$ given by $r /\left\|\left(x-x^{e}, u-u^{e}\right)\right\|$. Then, since $L(\bar{x}, \bar{u}) \leq \eta L(x, u)$ holds by convexity of $L$,

$$
L(x, u) \geq \frac{L(\bar{x}, \bar{u})}{\eta}=\frac{L(\bar{x}, \bar{u})}{r}\left\|\left(x-x^{e}, u-u^{e}\right)\right\| \geq(c / r) \cdot\left\|\left(x-x^{e}, u-u^{e}\right)\right\|
$$

follows. This implies $L(x, u) \rightarrow \infty$ for $\|(x, u)\| \rightarrow \infty$ and, thus, radial unboundedness of $L$.

If $\mathbb{Y}$ is bounded, [18, Lemma 4.3] only yields (4.10) with $\gamma \in \mathcal{K}$. However, since no conditions are imposed on $(r, \infty), r:=\max \{\|(x, u)\|:(x, u) \in \mathbb{Y}\}$, by $(4.10), \gamma$ can be modified such that $\gamma \in \mathcal{K}_{\infty}$ holds.

The particular form of $\alpha$ for $\ell$ from (4.3) follows from the fact that for $\ell$ as above the modified cost $\tilde{\ell}$ is again of the form (4.3) and attains its minimum at $\left(x^{e}, u^{e}\right)$. Hence $\tilde{\ell}$ is of the form

$$
\tilde{\ell}(x, u)=\left(x-x^{e}\right)^{T} R\left(x-x^{e}\right)+\left(u-u^{e}\right)^{T} Q\left(u-u^{e}\right)
$$

and since strict convexity of $\ell$ implies $R>0, \tilde{\ell}$ has the claimed quadratic lower bound.

$\square$

Proposition 4.3 is readily applicable to Example 2.4 , since $\ell$ in this example is of the form (4.3) with $R>0$ and $Q>0$ and the convex constraint set contains equilibrium pairs in its interior. A little computation shows that the optimal equilibrium pair is given by $x^{e} \approx(3.546,14.653)^{T}, u^{e} \approx 6.163$ and $\lambda$ in (4.1) is given by $\lambda(x)=\nu^{T} x$ with $\nu^{T} \approx(-368.6684,-503.5415)^{T}$. However, Proposition 4.3 does not apply to Example 2.3, since in this example the cost is of the form (4.3) with $R=0$ and $Q=1$, hence it is not strictly convex. 
In order to also cover this situation, we now investigate costs of the form (4.3) with $R \geq 0$. The next lemma shows that under a spectral condition on $A$ we can always turn such a cost into a strictly convex cost and the subsequent Proposition 4.5 shows that we can use this in order to obtain strict dissipativity.

LEMma 4.4. Consider the optimal control problem (2.3) with dynamics (4.2), cost function (4.3) with $R \geq 0$ and $Q>0$ and constraint set $\mathbb{Y}$ defined via (2.2) with a convex function $\mathrm{g}$. Assume that $\sigma(A) \cap \mathbb{C}_{=1}=\emptyset$. Then there exists a function $\lambda: \mathbb{R}^{n} \rightarrow \mathbb{R}$ of the form $\lambda(x)=x^{T} P x$ for a symmetric matrix $P \in \mathbb{R}^{n \times n}$ with $P>0$ such that the map

$$
(x, u) \mapsto \bar{\ell}(x, u):=\ell(x, u)+\lambda(x)-\lambda(A x+B u+c)
$$

is strictly convex and radially unbounded.

Proof. It is sufficient to show the claim for $\ell_{1}(x, u):=u^{T} Q u$, because if

$$
(x, u) \mapsto \bar{\ell}_{1}(x, u):=\ell_{1}(x, u)+\lambda(x)-\lambda(A x+B u+c)
$$

is strictly convex then $\bar{\ell}$ is strictly convex, too, because the difference $\bar{\ell}-\bar{\ell}_{1}=x^{T} R x+$ $s^{T} x+v^{T} u$ consists of linear terms and of a quadratic term which is (possibly non strictly) convex in $x$. Moreover, it is sufficient to show the assertion for $c=0$ in (4.2), because for $\lambda=x^{T} P x$ the difference $\lambda(A x+B u+c)-\lambda(A x+B u)$ is an affine linear function in $(x, u)$, hence the additional terms appearing in $\bar{\ell}_{1}$ for $c \neq 0$ will not affect convexity of $\bar{\ell}_{1}$.

For $\ell_{1}$, however, by Proposition 8.2 we can find $P>0$ such that

$$
(x, u) \mapsto u^{T} Q u+x^{T} P x-\left(x^{T} A^{T}+u^{T} B^{T}\right) P(A x+B u)
$$

is strictly convex. Hence, $\lambda(x)=x^{T} P x$ satisfies the assertion. $\square$

Proposition 4.5. Consider the optimal control problem with dynamics (4.2), cost function (4.3) with $R \geq 0$ and $Q>0$ and constraint set $\mathbb{Y}$ defined via (2.2) with a convex function $\mathrm{g}$. Assume that the problem (4.4) satisfies the Slater condition (4.5) and that either $R>0$ or $\sigma(A) \cap \mathbb{C}_{=1}=\emptyset$ holds. Then there exists $\nu \in \mathbb{R}^{n}$ and a symmetric matrix $P \in \mathbb{R}^{n \times n}$ with $P \geq 0$ such that the problem is strictly dissipative with $\lambda(x):=x^{T} P x+\nu^{T} x$. Moreover, the resulting modified stage cost $\tilde{\ell}$ is of the form (4.12) and $\alpha(r)=C r^{2}$ for some $C>0$.

Proof. In case $\sigma(A) \cap \mathbb{C}_{=1}=\emptyset$, by Lemma 4.4 we can find $P>0$ such that

$$
\bar{\ell}(x, u)=\ell(x, u)+x^{T} P x-(A x+B u+c)^{T} P(A x+B u+c)
$$

is strictly convex. Since the matrices in $\bar{\ell}$ are positive definite, the existence of a global minimum $\left(x^{e}, u^{e}\right)$ of (4.4) for $\ell=\bar{\ell}$ follows. Hence, Proposition 4.3 applies to $\bar{\ell}$ and implies that there exists $\nu \in \mathbb{R}^{n}$ such that

$$
\begin{aligned}
\tilde{\ell}(x, u) & =\bar{\ell}(x, u)+\nu^{T} x-\nu^{T}(A x+B u+c) \\
& =\ell(x, u)+\lambda(x)-\lambda(A x+B u+c)
\end{aligned}
$$

satisfies (4.1).

In case $R>0$ we can directly use Proposition 4.3 because existence of a global minumum $\left(x^{e}, u^{e}\right)$ of (4.4) follows from the positive definiteness of $R$ and $Q$. In this case we obtain $P=0$.

In both cases, the fact that $\tilde{\ell}$ is of the form (4.12) follows from the proof of Proposition 4.3 and implies the quadratic form of $\alpha$. 
Since we have $A=2$ in Example 2.3, the condition $\sigma(A) \cap \mathbb{C}_{=1}=\emptyset$ holds and we can apply Proposition 4.5 to this example. One easily checks that (4.1) holds with a quadratic $\alpha$, e.g., for $\lambda(x)=-x^{2} / 2$.

We remark that strict dissipativity together with suitable controllability and boundedness conditions implies the turnpike property, see [13, Theorem 5.3]. However, the turnpike property derived in this reference will in general not be exponential. Hence, additional conditions are needed in order to obtain the exponential property and this is what we will investigate in the subsequent sections.

5. A value function condition. In this section we derive a condition for the exponential turnpike property for strictly dissipative systems by means of an auxiliary optimal control problem. This condition is implicit in the sense that it requires knowledge of bounds for the optimal value function of this auxiliary problem. Checkable sufficient conditions for the existence of these bounds based on the original problem (2.3) and the system dynamics are discussed in the subsequent section.

In order to formulate our criterion, using $\tilde{\ell}$ from (4.1) we define the modified optimal control problem

$$
\text { minimize } \widetilde{J}_{N}(x, u):=\sum_{k=0}^{N-1} \tilde{\ell}\left(x_{u}(k, x), u(k)\right) \quad \text { over } \quad u \in \mathcal{U}^{N}(x) .
$$

Note that $\widetilde{J}_{N}$ and $J_{N}$ from (2.3) are related via

$$
\widetilde{J}_{N}(x, u)=J_{N}(x, u)+\lambda(x)-\lambda\left(x_{u}(N, x)\right) .
$$

The auxiliary optimal value function needed for our criterion is obtained from the following terminal constrained optimal control problem.

Definition 5.1. For each $N \in \mathbb{N}$ and each two points $x, \bar{x} \in \mathbb{X}$ for which an admissible trajectory with $x_{u}(N, x)=\bar{x}$ exists, we define the optimal value function

$$
\widetilde{V}_{N}(x, \bar{x})=\inf _{u \in \mathcal{U}^{N}(x): x_{u}(N, x)=\bar{x}} \widetilde{J}_{N}(x, u) .
$$

Due to (5.2) it follows that the optimal trajectory for the optimal control problem from Definition 5.1 does not change if we replace $\widetilde{J}_{N}$ by $J_{N}$. Particularly, if we pick the optimal control sequence $u_{x, N}^{\star}$ from (2.5), then $u_{x, N}^{\star}$ is also an optimal control sequence for the problem from Definition 5.1 with $\bar{x}:=x_{u_{x, N}^{\star}}(N)$.

Throughout this section we assume that $\ell\left(x^{e}, u^{e}\right)=\tilde{\ell}\left(x^{e}, u^{e}\right)=0$ holds. Recall that this condition can be assumed without loss of generality, see Remark 4.2. Moreover, we define

$$
\ell^{\star}(x):=\min _{u \in \mathbb{U}(x)} \ell(x, u) \quad \text { and } \quad \tilde{\ell}^{\star}(x):=\min _{u \in \mathbb{U}(x)} \tilde{\ell}(x, u) .
$$

Note that dissipativity together with $\tilde{\ell}\left(x^{e}, u^{e}\right)=0$ implies $\tilde{\ell}^{\star}(x) \geq \alpha\left(|x|_{x^{e}}\right)$.

The condition in order to ensure the exponential turnpike property is the following bound on $\widetilde{V}_{N}(x, \bar{x})$ from Definition 5.1.

Assumption 5.2. Let $\bar{N} \geq 1$ and consider sets $\mathbb{Z}_{N} \subseteq \mathbb{X} \times \mathbb{X}$ for $N \geq \bar{N}$ such that for all $(x, \bar{x}) \in \mathbb{Z}_{N}$ there exists an admissible trajectory with $x_{u}(N, x)=\bar{x}$. We assume that for all $N \geq \bar{N}$ there exist $\gamma_{N}, \delta_{N} \geq 1$ such that for all $(x, \bar{x}) \in \mathbb{Z}_{N}$ the inequality

$$
\widetilde{V}_{N}(x, \bar{x}) \leq \gamma_{N} \tilde{\ell}^{\star}(x)+\left(\delta_{N}-1\right) \tilde{\ell}^{\star}(\bar{x})
$$


holds.

In order to prove the exponential turnpike property we need the following preparatory lemma.

Lemma 5.3. Let $k \in \mathbb{N}$ and real values $\ell_{0}, \ell_{1}, \ldots, \ell_{k}, \ell_{0}^{\star}, \ell_{1}^{\star}, \ldots, \ell_{k}^{\star}$ with $0 \leq \ell_{k}^{\star} \leq$ $\ell_{k}$, and $\gamma_{1}, \gamma_{2}, \ldots, \gamma_{k} \geq 1$ be given such that the condition

$$
\sum_{j=m}^{k} \ell_{j} \leq \gamma_{k-m} \ell_{m}^{\star}, \quad m=0,1, \ldots, k-1
$$

is satisfied. Then the following estimate holds

$$
\ell_{k} \leq\left(\gamma_{k}-1\right)\left(\prod_{m=1}^{k-1} \frac{\gamma_{m}-1}{\gamma_{m}}\right) \ell_{0}^{\star}
$$

Proof. If an index $m \in\{0,1,2, \ldots, k-1\}$ exists such that $\gamma_{k-m}=1$ holds, then $\ell_{m+1}=\ldots=\ell_{k}=0$ immediately follows from (5.5) and the assertion holds. Hence, suppose that the condition $\gamma_{k-m}>1$ is satisfied for each $m \in\{0,1,2, \ldots, k-1\}$. From $\ell_{m}^{\star} \leq \ell_{m}$ and (5.5) we obtain

$$
\sum_{j=m+1}^{k} \ell_{j} \leq\left(\gamma_{k-m}-1\right) \ell_{m}^{\star}, \quad m=0,1, \ldots, k-1 .
$$

Using $\ell_{m}^{\star} \leq \ell_{m}$ and Inequality (5.7) yields

$$
\ell_{m}+\sum_{j=m+1}^{k} \ell_{j} \geq \frac{\sum_{j=m+1}^{k} \ell_{j}}{\gamma_{k-m}-1}+\sum_{j=m+1}^{k} \ell_{j}=\frac{\gamma_{k-m}}{\gamma_{k-m}-1}\left(\ell_{m+1}+\sum_{j=m+2}^{k} \ell_{j}\right)
$$

for each $m \in\{0,1,2, \ldots, k-1\}$. Using this inequality inductively for $m=1, \ldots, k-1$ yields

$$
\ell_{1}+\sum_{j=2}^{k} \ell_{j} \geq\left(\prod_{m=1}^{k-1} \frac{\gamma_{k-m}}{\gamma_{k-m}-1}\right) \ell_{k}
$$

Applying (5.7) once more for $m=0$ and renumbering the indices of the $\gamma_{k-m}$ leads to

$$
\left(\gamma_{k}-1\right) \ell_{0}^{\star} \geq \ell_{1}+\sum_{j=2}^{k} \ell_{j} \geq\left(\prod_{m=1}^{k-1} \frac{\gamma_{m}}{\gamma_{m}-1}\right) \ell_{k}
$$

and reordering the factors in this inequality completes the proof. $\square$

This lemma is the key ingredient in order to prove the following proposition in which we provide an exponentially decaying upper bound for the modified stage cost along an optimal trajectory for the original optimal control problem (2.3). The exponential turnpike property will be concluded from this estimate in the subsequent theorem.

Proposition 5.4. Consider a dissipative optimal control problem (2.3) and the modified cost $\tilde{\ell}$ from (4.1), assuming without loss of generality $\tilde{\ell}\left(x^{e}, u^{e}\right)=0$. Let $\mathbb{X}_{0} \subseteq \mathbb{X}$ be a set of initial values such that $V_{N}(x)<\infty$ holds for all $x \in \mathbb{X}_{0}$ and all 
$N \in \mathbb{N}$. For each $x \in \mathbb{X}_{0}$ and $N \in \mathbb{N}$ consider the optimal trajectories $x_{u_{x, N}^{\star}}(\cdot, x)$ of (2.3) and define the sets

$$
\mathbb{Z}_{N}:=\left\{\begin{array}{l|l}
\left(x_{u_{x, \widetilde{N}}^{\star}}(k, x), x_{u_{x, \widetilde{N}}^{\star}}(k+N, x)\right) \mid \begin{array}{l}
x \in \mathbb{X}_{0}, \tilde{N} \in \mathbb{N}, \widetilde{N} \geq N \\
k=0, \ldots, \widetilde{N}-N
\end{array}
\end{array}\right\} .
$$

Suppose that Assumption 5.2 holds for some $\bar{N} \geq 1$ with $\mathbb{Z}_{N}$ from (5.8) and $\gamma_{N}$ and $\delta_{N}$ decreasing in $N$. Then, for each optimal trajectory $x_{u_{x, N}^{\star}}(\cdot, x)$ with $x \in \mathbb{X}_{0}$ there exists $N^{\prime} \in\{1, \ldots, N\}$ such that

$$
\tilde{\ell}\left(x_{u_{x, N}^{\star}}(k, x), u_{x, N}^{\star}(k)\right) \leq\left(\gamma_{k}-1\right)\left(\prod_{m=1}^{k-1} \frac{\gamma_{m}-1}{\gamma_{m}}\right) \tilde{\ell}^{\star}(x)
$$

holds for all $k=1,2, \ldots, N^{\prime}-1$ and

$$
\tilde{\ell}\left(x_{u_{x, N}^{\star}}(k, x), u_{x, N}^{\star}(k)\right) \leq\left(\delta_{N-k}-1\right)\left(\prod_{m=1}^{N-k-1} \frac{\delta_{m}-1}{\delta_{m}}\right) \tilde{\ell}^{\star}\left(x_{u_{x, N}^{\star}}(N, x)\right)
$$

holds for all $k=\max \left\{N^{\prime}+\bar{N}-2, N^{\prime}+1\right\}, \ldots, N-1$.

Proof. We use the abbreviations

$$
\tilde{\ell}_{k}:=\tilde{\ell}\left(x_{u_{x, N}^{\star}}(k ; x), u_{x, N}^{\star}(k)\right) \quad \text { and } \quad \tilde{\ell}_{k}^{\star}:=\tilde{\ell}^{\star}\left(x_{u_{x, N}^{\star}}(k, x)\right)
$$

for $k=0,1, \ldots, N-1$ and for $k=N$ we set $\tilde{\ell}_{N}:=\tilde{\ell}_{N}^{\star}:=\tilde{\ell}^{\star}\left(x_{u_{x, N}^{\star}}(N, x)\right)$. Then, we choose $N^{\prime} \in\{1, \ldots, N\}$ maximal such that

$$
\sum_{j=m}^{N^{\prime}-1} \tilde{\ell}_{j} \leq \gamma_{N^{\prime}-1-m} \tilde{\ell}_{m}^{\star} \quad \text { holds for all } m=0,1, \ldots, N^{\prime}-2 .
$$

Note that this condition is trivially satisfied for $N^{\prime}=1$, hence we get $N^{\prime} \geq 1$. For each $k=1,2, \ldots, N^{\prime}-1$ Inequality (5.11), the nonnegativity of the $\tilde{\ell}_{j}$ and the monotonicity of the $\gamma_{N}$ imply

$$
\sum_{j=m}^{k} \tilde{\ell}_{j} \leq \sum_{j=m}^{N^{\prime}-1} \tilde{\ell}_{j} \leq \gamma_{N^{\prime}-1-m} \tilde{\ell}_{m}^{\star} \leq \gamma_{k-m} \tilde{\ell}_{m}^{\star} \quad \text { for all } m=0,1, \ldots, k-1 .
$$

Hence, we can apply Lemma 5.3 for each $k=1,2, \ldots, N^{\prime}-1$ with $\ell_{j}=\tilde{\ell}_{j}$ and $\ell_{j}^{\star}=\tilde{\ell}_{j}^{\star}$ which yields Inequality (5.9).

It remains to show Inequality (5.10) provided $N^{\prime}+\bar{N} \leq N+1$. To this end, observe that the definition of $N^{\prime}$ via (5.11) implies the existence of an index $m^{\prime} \in$ $\left\{0,1, \ldots, N^{\prime}-1\right\}$ such that

$$
\sum_{j=m^{\prime}}^{N^{\prime}} \tilde{\ell}_{j}>\gamma_{N^{\prime}-m^{\prime}} \tilde{\ell}_{m^{\prime}}^{\star}
$$

holds. Now observe that the restriction of $x_{u_{x, N}^{\star}}(\cdot, x)$ to $\left\{m^{\prime}, \ldots, N-m\right\}$ is again an optimal trajectory for the terminal constrained problem from Definition 5.1. For 
$m=0,1, \ldots, N-\bar{N}-N^{\prime}+1$, from (5.4) we can thus conclude

$$
\begin{aligned}
\sum_{j=m^{\prime}}^{N-m} \tilde{\ell}_{j} & =\sum_{j=m^{\prime}}^{N-m-1} \tilde{\ell}_{j}+\tilde{\ell}_{N-m} \\
& \leq \gamma_{N-m-m^{\prime}} \tilde{\ell}_{m^{\prime}}^{\star}+\left(\delta_{N-m-m^{\prime}}-1\right) \tilde{\ell}_{N-m}^{\star}+\tilde{\ell}_{N-m} \\
& \leq \gamma_{N-m-m^{\prime}} \tilde{\ell}_{m^{\prime}}+\delta_{N-m-m^{\prime}} \tilde{\ell}_{N-m} .
\end{aligned}
$$

From this inequality and (5.12) we obtain

$$
\sum_{j=N^{\prime}+1}^{N-m} \tilde{\ell}_{j}=\sum_{j=m^{\prime}}^{N-m} \tilde{\ell}_{j}-\sum_{j=m^{\prime}}^{N^{\prime}} \tilde{\ell}_{j} \leq \gamma_{N-m-m^{\prime}} \tilde{\ell}_{m^{\prime}}+\delta_{N-m-m^{\prime}} \tilde{\ell}_{N-m}-\gamma_{N^{\prime}-m^{\prime}} \tilde{\ell}_{m^{\prime}}^{\star}
$$

for $m=0,1, \ldots, N-\bar{N}-N^{\prime}+1$. Since $\gamma_{N}$ is decreasing in $N$ and $N-m \geq \bar{N}+N^{\prime}-1 \geq$ $N^{\prime}$ we obtain $\gamma_{N-m-m^{\prime}} \leq \gamma_{N^{\prime}-m^{\prime}}$ and thus

$$
\sum_{j=N^{\prime}+1}^{N-m} \tilde{\ell}_{j} \leq \delta_{N-m-m^{\prime}} \tilde{\ell}_{N-m}
$$

for $m=0,1, \ldots, N-\bar{N}-N^{\prime}+1$. Changing the summation index from $j$ to $N-j$ and using that $\delta_{N}$ is also decreasing in $N$ and $-m^{\prime} \geq-N^{\prime}-1$ we can continue

$$
\sum_{j=m}^{N-N^{\prime}-1} \tilde{\ell}_{N-j} \leq \delta_{N-m-m^{\prime}} \tilde{\ell}_{N-m} \leq \delta_{N-N^{\prime}-1-m} \tilde{\ell}_{N-m}
$$

Now for each $k=1, \ldots, \min \left\{N-N^{\prime}-\bar{N}+2, N-N^{\prime}-1\right\}$ we obtain

$$
\sum_{j=m}^{k} \tilde{\ell}_{N-j} \leq \sum_{j=m}^{N-N^{\prime}-1} \tilde{\ell}_{N-j} \leq \delta_{N-N^{\prime}-1-m} \tilde{\ell}_{N-m} \leq \delta_{k-m} \tilde{\ell}_{N-m}
$$

for all $m=0, \ldots, k-1$. Hence, for all these $k$ we can apply Lemma 5.3 with $\ell_{j}=$ $\ell_{j}^{\star}=\tilde{\ell}_{N-j}$ and $\gamma_{j}=\delta_{j}$. This implies

$$
\tilde{\ell}_{N-k} \leq\left(\delta_{k}-1\right)\left(\prod_{m=1}^{k-1} \frac{\delta_{m}-1}{\delta_{m}}\right) \tilde{\ell}_{N}=\left(\delta_{k}-1\right)\left(\prod_{m=1}^{k-1} \frac{\delta_{m}-1}{\delta_{m}}\right) \tilde{\ell}_{N}^{\star}
$$

for $k=1, \ldots, \min \left\{N-N^{\prime}-\bar{N}+2, N-N^{\prime}-1\right\}$ which is equivalent to (5.10). $\square$

REMARK 5.5. The assumptions of Proposition 5.4 imply the inequalities $\gamma_{N} \leq$ $\gamma_{1}=: \gamma$ and $\delta_{N} \leq \delta_{1}=: \delta$ for all $N \geq 1$. These inequalities imply that Proposition 5.4 yields the estimates

$$
\tilde{\ell}\left(x_{u_{x}^{\star}}(k, x), u_{x}^{\star}(k)\right) \leq \gamma \mu^{k} \tilde{\ell}^{\star}(x)
$$

for $\mu=(\gamma-1) / \gamma \in(0,1)$ and all $k=1,2, \ldots, N^{\prime}-1$ and

$$
\tilde{\ell}\left(x_{u_{x}^{\star}}(k, x), u_{x}^{\star}(k)\right) \leq \delta \nu^{N-k} \tilde{\ell}^{\star}\left(x_{u_{x}^{\star}}(N, x)\right)
$$

for $\nu=(\delta-1) / \delta \in(0,1)$ and all $k=\max \left\{N^{\prime}+\bar{N}-2, N^{\prime}+1\right\}, \ldots, N-1$. 
Now we can state our main theorem which derives the exponential turnpike property from Assumption 5.2.

THEOREM 5.6. Consider a strictly dissipative optimal control problem (2.3) and the modified stage cost $\tilde{\ell}$ from (4.1), assuming without loss of generality $\tilde{\ell}\left(x^{e}, u^{e}\right)=0$. Consider a set of initial values $\mathbb{X}_{0} \subseteq \mathbb{X}$ such that $V_{N}(x)<\infty$ holds for all $x \in \mathbb{X}_{0}$ and all $N \in \mathbb{N}$ and suppose that the following conditions hold.

(i) Inequality (4.1) holds with

$$
\alpha(r) \geq M \min \left\{r^{p}, r^{q}\right\}
$$

for positive constants $M, p, q \in \mathbb{R}$ and all $r \geq 0$.

(ii) Assumption 5.2 holds for some $\bar{N} \geq 1$ with $\gamma_{N}$ and $\delta_{N}$ which are decreasing in $N$ on the sets $\mathbb{Z}_{N}$ from (5.8).

(iii-a) Either $\tilde{\ell}^{\star}$ is bounded on $\mathbb{X}$ or

(iii-b) $\sup _{x \in \mathbb{X}_{0}, N \in \mathbb{N}} V_{N}(x)<\infty, \tilde{\ell}^{\star}$ is bounded on $\mathbb{X}_{0}$ and on any bounded subset of $\mathbb{X}$ and $\ell^{\star}$ is bounded from below and radially unbounded on $\mathbb{X}$.

Then the system has the exponential turnpike property from Definition 2.2(ii).

Proof. The assumptions of the theorem include those of Proposition 5.4 which implies the Inequalities (5.13) and (5.14). From these inequalities we obtain the estimate

$$
\tilde{\ell}\left(x_{u_{x, N}^{\star}}(k, x), u_{x, N}^{\star}(k)\right) \leq \begin{cases}K_{1}(x) \eta^{k}, & k=0, \ldots, N^{\prime}-1 \\ K_{2}(x, N) \eta^{N-k}, & k=\max \left\{N^{\prime}+\bar{N}-2, N^{\prime}+1\right\}, \ldots, N\end{cases}
$$

for $\eta=\max \{\mu, \nu\} \in(0,1)$ and

$$
K_{1}(x):=\gamma \tilde{\ell}^{\star}(x), \quad K_{2}(x, N):=\delta \tilde{\ell}^{\star}\left(x_{u_{x, N}^{\star}}(N, x)\right) .
$$

If case (iii-a) holds, by setting $K:=\max \{\gamma, \delta\} \max _{x^{\prime} \in \mathbb{X}} \tilde{\ell}^{\star}\left(x^{\prime}\right)$ we obtain

$$
\max \left\{K_{1}(x), K_{2}(x, N)\right\} \leq K \quad \text { for all } x \in \mathbb{X}_{0}, N \in \mathbb{N} .
$$

If case (iii-b) holds, $K_{1}(x)$ can be bounded by $\gamma \max _{x^{\prime} \in \mathbb{X}_{0}} \tilde{\ell}^{\star}\left(x^{\prime}\right)$. Since $V_{N}$ is bounded on $\mathbb{X}_{0}$ uniformly in $N$ and $\ell^{\star}$ is bounded from below on $\mathbb{X}$, we obtain uniform boundedness of $\ell^{\star}\left(x_{u_{x, N}^{\star}}(N, x)\right)$ in $x \in \mathbb{X}_{0}$ and $N$. Hence, since $\ell^{\star}$ is radially unbounded, there exists a bounded set $\mathbb{X}_{1} \subset \mathbb{X}$ with $x_{u_{x, N}^{\star}}(N, x) \in \mathbb{X}_{0}$ and since $\tilde{\ell}^{\star}$ is bounded on the bounded set $\mathbb{X}_{1}$ the expression $K_{2}(x, N)$ is bounded from above for all $x \in \mathbb{X}_{0}$ and $N \in \mathbb{N}$. Hence, also in case (iii-b) we can find $K>0$ such that (5.16) holds.

Now observe that the set of $k$ for which neither inequality in (5.15) holds has at $\operatorname{most} \widetilde{N}:=\max \{1, \bar{N}-2\}$ elements. For any $P \in \mathbb{N}$ and $N \geq P+\widetilde{N}$ we define the number $R:=\lfloor(N-\widetilde{N}-P+1) / 2\rfloor$ and the set

$$
\mathcal{Q}:=\left\{R, \ldots, N^{\prime}-1, \max \left\{N^{\prime}+\bar{N}-2, N^{\prime}+1\right\}, \ldots, N-R\right\} .
$$

This set has at least

$$
\# \mathcal{Q}=N-2 R+1-\widetilde{N} \geq N-(N-\widetilde{N}-P+1)+1-\widetilde{N}=P
$$

elements and for all $k \in \mathcal{Q}$ we have $k \geq R$ and $N-k \geq R$ which implies

$$
\tilde{\ell}\left(x_{u_{x, N}^{\star}}(k, x), u_{x}^{\star}(k)\right) \leq K \eta^{R} \quad \text { for all } k \in \mathcal{Q} .
$$


Since $R \geq(N-\widetilde{N}-P) / 2$ and $\eta \in(0,1)$ we obtain

$$
K \eta^{R} \leq K \eta^{(N-\widetilde{N}-P) / 2}=K \eta^{(-\widetilde{N}-P) / 2}\left(\eta^{1 / 2}\right)^{N} .
$$

Now the lower bound on $\alpha_{\ell}$ implies $\alpha_{\ell}^{-1}(s) \leq \max \left\{s^{1 / p}, s^{1 / q}\right\} / M$. Assuming without loss of generality $p \geq q$ and $K \eta^{(-\widetilde{N}-P) / 2} \geq 1$ we can conclude

$$
\left|x_{u_{x}^{\star}}(k, x)\right|_{x^{e}} \leq \alpha_{\ell}^{-1}\left(\tilde{\ell}\left(x_{u_{x}^{\star}}(k, x), u_{x}^{\star}(k)\right)\right) \leq\left(K \eta^{(-\widetilde{N}-P) / 2}\right)^{1 / q}\left(\eta^{1 /(2 p)}\right)^{N} / M
$$

for all $k \in \mathcal{Q}$. This implies that $\sigma_{P}$ can be chosen of the exponential form from Definition 2.2(ii) with $C_{P}=\left(K \eta^{(-\widetilde{N}-P) / 2}\right)^{1 / q} / M$ and $\theta=\eta^{1 /(2 p)}$.

6. Conditions on the dynamics. The conditions of Theorem 5.6 are difficult to check since they rely on bounds on the auxiliary value function $\widetilde{V}_{N}(x, \bar{x})$ on the implicitly defined sets $\mathbb{Z}_{N}$. In this section we present two results in which we give sufficient conditions in terms of systems theoretic properties of $f$. The results cover two "extreme" cases, one for strictly convex affine linear quadratic problems without constraints, in which we will see that the exponential turnpike property is equivalent to the stabilizability of the pair $(A, B)$. The second case covers problems with (possibly) nonlinear dynamics and compact constraint set $\mathbb{Y}$, where we will see that controllability to a neighborhood of $x^{e}$ and a local controllability property around $x^{e}$ are sufficient in order to conclude the exponential turnpike property. These two cases should be seen as example results which illustrate how the assumptions of Theorem 5.6 can be verified by imposing structural assumptions on $f$ and $\ell$. Certainly, there are many other settings which can be treated with similar techniques.

In both cases, we start with a proposition which proves Assumption 5.2 and then formulate a theorem which proves the exponential turnpike property. We start with the affine linear quadratic case without constraints.

Proposition 6.1. Consider the optimal control problem (2.3) with dynamics (4.2) and cost function (4.3) with $R \geq 0$ and $Q>0$. Let $\mathbb{Y}=\mathbb{R}^{n} \times \mathbb{R}^{m}$ and assume that the conditions of Proposition 4.5 hold and that the pair $(A, B)$ is stabilizable. Then there exist $\gamma>0$ and $\delta>0$ such that Assumption 5.2 holds for $\bar{N}=n$ and $\gamma_{N}=\gamma, \delta_{N}=\delta$ on

$$
\mathbb{Z}_{N}=\left\{\left(x_{1}, x_{2}\right) \in \mathbb{R}^{n} \times \mathbb{R}^{n} \mid \text { there exists } u \in\left(\mathbb{R}^{m}\right)^{N} \text { with } x_{u}\left(N, x_{1}\right)=x_{2}\right\} .
$$

Proof. Performing the coordinate transformations $x:=x-x^{e}$ and $u:=u-u^{e}$ we may assume without loss of generality $x^{e}=0$ and $u^{e}=0$. Note that this implies $c=0$ in (4.2). Moreover, since the pair $(A, B)$ is stabilizable, applying a further linear coordinate transformation on $\mathbb{R}^{n}$, we may assume that the system matrices are in the form

$$
A=\left(\begin{array}{cc}
A_{1} & A_{2} \\
0 & A_{3}
\end{array}\right), \quad B=\left(\begin{array}{c}
B_{1} \\
0
\end{array}\right)
$$

where the pair $\left(A_{1}, B_{1}\right)$ is controllable and $\sigma\left(A_{3}\right) \subset \mathbb{C}_{<1}$, see [25, Lemma 3.3.3]. Splitting the state vector $x=\left(y^{T}, z^{T}\right)^{T}$ according to the splitting of $A$, the solutions of the system are of the form

$$
x_{u}\left(k, x_{0}\right)=\left(\begin{array}{c}
A_{1}^{k} y_{0}+R_{k} u_{k}+\sum_{j=0}^{k-1} A_{1}^{k-j-1} A_{2} A_{3}^{j} z_{0} \\
A_{3}^{k} z_{0}
\end{array}\right)
$$


where $R_{k}=\left(B_{1}, A_{1} B_{1}, \ldots, A_{1}^{k-1} B_{1}\right)$ and $u_{k}=\left(u(0)^{T}, \ldots, u(k-1)^{T}\right)^{T}$.

Now consider $\left(x_{1}, x_{2}\right) \in \mathbb{Z}_{n}$ and denote by $\tilde{u}(\cdot)$ the control sequence satisfying $x_{\tilde{u}}\left(n, x_{1}\right)=x_{2}$. Writing $x_{1}=\left(y_{1}^{T}, z_{1}^{T}\right), x_{2}=\left(y_{2}^{T}, z_{2}^{T}\right)^{T}$, (6.1) implies $z_{2}=A_{3}^{n} z_{1}$. Moreover, since $\left(A_{1}, B_{1}\right)$ is controllable, the matrix $R_{n}$ has full rank and hence $u_{n}=$ $R_{n}^{-1}\left(y_{2}-A_{1}^{n} y_{1}-\sum_{j=0}^{n-1} A_{1}^{n-j-1} A_{2} A_{3}^{j} z_{1}\right) \in \mathbb{R}^{m n}$ is well defined and has a norm bounded by $C\left(\left\|x_{1}\right\|+\left\|x_{2}\right\|\right)$ for some appropriate constant $C>0$ independent of $x_{1}$ and $x_{2}$ (for details see also the corresponding continuous time computation in [25, Section 3.5]). Denoting the control sequence of length $n$ corresponding to $u_{n}$ by $u$, this yields a control satisfying $x_{u}\left(n, x_{1}\right)=x_{2}$,

$$
\left\|x_{u}\left(k, x_{1}\right)\right\| \leq C_{1}\left(\left\|x_{1}\right\|+\left\|x_{2}\right\|\right) \quad \text { and } \quad\|u(k)\| \leq C_{1}\left(\left\|x_{1}\right\|+\left\|x_{2}\right\|\right)
$$

for all $k=0, \ldots, n-1$ and an appropriate constant $C_{1}$ independent of $x_{1}$ and $x_{2}$.

Now consider $(x, \bar{x}) \in \mathbb{Z}_{N}$ for some $N \geq n$. Since the system is stabilizable, there exists $C_{2}>0$ and $\sigma \in(0,1)$ (independent of $x$ ) such that we can find a control sequence $\bar{u}$ with

$$
\left\|x_{\bar{u}}(k, x)\right\| \leq C_{2} \sigma^{k}\|x\| \quad \text { and } \quad\|\bar{u}(k)\| \leq C_{2} \sigma^{k}\|x\|
$$

for all $k \geq 0$. We apply this control for $k=0, \ldots, N-n$ and then apply $u$ from (6.2) for $x_{1}=x_{\bar{u}}(N-n, x)$ and $x_{2}=\bar{x}$, using that the structure of the solution from (6.1) implies that there exists a control $\tilde{u}$ with $x_{\tilde{u}}\left(n, x_{1}\right)=x_{2}$, i.e., that $\left(x_{1}, x_{2}\right) \in \mathbb{Z}_{n}$. Denoting the resulting control by $\hat{u}$ we obtain a trajectory satisfying $x_{\hat{u}}(N, x)=\bar{x}$. Observing that (4.12) implies $\tilde{\ell}(x, u) \leq C_{3}\left(\|x\|^{2}+\|u\|^{2}\right)$ for some suitable $C_{3}>0$ (recall that we assumed $x^{e}=0$ and $u^{e}=0$ ) we obtain

$$
\begin{aligned}
\sum_{k=0}^{N-1} \tilde{\ell}\left(x_{\hat{u}}(k, x), \hat{u}(k)\right) & =\sum_{k=0}^{N-n-1} \tilde{\ell}\left(x_{\bar{u}}(k, x), \bar{u}(k)\right)+\sum_{k=0}^{n-1} \tilde{\ell}\left(x_{u}\left(k, x_{1}\right), u(k)\right) \\
& \leq\left(\sum_{k=0}^{N-n-1} 2 C_{2}^{2} C_{3} \sigma^{2 k}\|x\|^{2}\right)+2 n C_{1}^{2} C_{3}\left(C_{2} \sigma^{(N-n)}\|x\|+\|\bar{x}\|\right)^{2} \\
& \leq\left(2 C_{2}^{2} C_{3} \frac{1}{1-\sigma^{2}}+4 n C_{1}^{2} C_{2}^{2} C_{3}\right)\|x\|^{2}+4 n C_{1}^{2} C_{3}\|\bar{x}\|^{2} \\
& \leq \gamma \tilde{\ell}^{*}(x)+(\delta-1) \tilde{\ell}^{*}(\bar{x})
\end{aligned}
$$

for suitable $\gamma, \delta>0$ since $\tilde{\ell}^{*}(x)$ is bounded from below by $\eta\|x\|^{2}$ for some $\eta>0$. This implies the desired inequality for $\widetilde{V}_{N}$.

Using this proposition we can derive the following equivalence result between the stabilizability of $(A, B)$ and the exponential turnpike property.

TheOREM 6.2. Consider the optimal control problem (2.3) with dynamics (4.2) and cost function (4.3) with $R>0$ and $Q>0$. Let $\mathbb{Y}=\mathbb{R}^{n} \times \mathbb{R}^{m}$. Then there exists an equilibrium $x^{e} \in \mathbb{R}^{n}$ such that for each compact subset $\mathbb{X}_{0} \subset \mathbb{R}^{n}$ the system has the exponential turnpike property if and only if the pair $(A, B)$ is stabilizable.

Proof. Assume that there exists an equilibrium $x^{e}$ such that the system has the exponential turnpike property on each compact subset $\mathbb{X}_{0} \subset \mathbb{R}^{n}$. This implies that for each initial value $x \in \mathbb{R}^{n}$ and each neighborhood $\mathcal{N}$ of $x^{e}$ we can control the system from $x$ to $\mathcal{N}$. This implies stabilizability of $(A, B)$.

Conversely, let $(A, B)$ be stabilizable. We check that the assumptions of Theorem 5.6 hold for each compact set $\mathbb{X}_{0} \subset \mathbb{R}^{n}$. Since $(A, B)$ is stabilizable, there exists a matrix $K$ with $\sigma(A-B K) \subset \mathbb{C}_{<1}$ and thus we obtain that there exists the equilibrium 
pair $(x, u)$ with $x=(\mathbb{I}-A+B K)^{-1} c$ and $u=B K x$ in $\mathbb{Y}$. Since $\ell$ is strictly convex and radially unbounded, (4.4) (with the trivial choice $g(x, u) \equiv-1$ ) has a global minimum $\left(x^{e}, u^{e}\right)$. Hence, we can apply Proposition 4.3 to conclude the existence of an equilibrium $x^{e}$ such that the system is strictly dissipative with $\alpha(r)=C r^{2}$ for some $C>0$, implying Condition (i) of Theorem 5.6 with $M=C$ and $p=q=2$.

Since $R>0$ the assumptions of Proposition 4.5 hold and thus also the assumptions of Proposition 6.1 hold, which implies Condition (ii) of Theorem 5.6.

Since $\mathbb{X}=\mathbb{R}^{n}$ and $\alpha$ in (4.1) is a $\mathcal{K}_{\infty}$-function, Condition (iii-a) of Theorem 5.6 cannot be satisfied. Hence, we verify Condition (iii-b). Clearly, as linear-quadratic functions $\tilde{\ell}^{\star}$ and $\ell^{\star}$ satisfy the properties from this condition. In order to see that $V_{N}$ is bounded on $\mathbb{X}_{0}$ independent of $N$, consider $x \in \mathbb{X}_{0}$ and the control function $\bar{u}$ from the proof of Proposition 6.1. Since $\ell$ is of the form (4.3) and we assume $\ell\left(x^{e}, u^{e}\right)=0$, we obtain that there exists $C>0$ such that $\ell(x, u) \leq C\left(\left\|x-x^{e}\right\|+\left\|u-u^{e}\right\|+1\right)(\| x-$ $\left.x^{e}\|+\| u-u^{e} \|\right)$. With a similar computation as in the proof of Proposition 6.1 we obtain

$$
V_{N}(x) \leq \sum_{k=0}^{N-1} \ell\left(x_{\bar{u}}(k, x), \bar{u}(k)\right) \leq C_{1}\left(\left\|x-x^{e}\right\|+\left\|x-x^{e}\right\|^{2}\right)
$$

for a suitable constant $C_{1}>0$. This shows that $V_{N}$ is bounded independent of $N$ on $\mathbb{X}_{0}$. Hence, all assumptions of Theorem 5.6 hold and thus the exponential turnpike property follows. $\mathrm{C}$

REMARK 6.3. (i) The constant $C_{P}$ resulting from the proof of Theorem 5.6 depends on the choice of $\mathbb{X}_{0}$. An inspection of the constants in this proof under the assumptions of Theorem 6.2 reveals that $C_{P} \leq M_{P} \max _{x \in \mathbb{X}_{0}}|x|_{x^{e}}$ for a constant $M_{P}$ independent of $\mathbb{X}_{0}$. Hence, $C_{P}$ grows linearly with the distance of the points in $\mathbb{X}_{0}$ from $x^{e}$.

(ii) We consider Theorem 6.2 remarkable since many known turnpike theorems (both exponential ones like [23, Theorem 2.3] and non exponential ones like [9, Theorem 4.2]), when specialized to the linear quadratic case require controllability of $(A, B)$ instead of the weaker stabilizability our theorem requires. The equivalence statement of our theorem moreover shows that one cannot further weaken this assumption.

Since the constraint sets in Example 2.4 are so large that they do not affect the optimal trajectories, Theorem 6.2 explains why we observe the exponential turnpike property in this example, since the pair $(A, B)$ in Example 2.4 is stabilizable (but not controllable). However, Theorem 6.2 is not applicable to Example 2.3 since the matrix $R$ in this example is not positive definite. Indeed, it is easy to see that the turnpike property does not hold for Example 2.3 if we choose $\mathbb{X}=\mathbb{R}$, since in this case $u_{x, N}^{\star} \equiv 0$ would be the optimal control for all initial values $x \in \mathbb{R}$ and all $N \in \mathbb{N}$ and thus the optimal solutions would never approach $x^{e}=0$ for $x \neq 0$. Consequently, compactness of $\mathbb{X}$ is crucial in this example and this is the second situation we investigate.

More precisely, we investigate conditions for the exponential turnpike property for possibly nonlinear dynamics and compact constraint set $\mathbb{Y}$. As a preparation for the subsequent Theorem 6.5, the following proposition shows that in this situation Assumption 5.2 holds on any set $\mathbb{X}_{0}$ if it holds locally around $x^{e}$ and if a boundedness condition on $V_{N}$ holds on $\mathbb{X}_{0}$

Proposition 6.4. Consider a strictly dissipative optimal control problem (2.3), assuming without loss of generality $\ell\left(x^{e}, u^{e}\right)=0$ and $\lambda\left(x^{e}\right)=0$. Assume that $\mathbb{X}$ is compact and that $f, \ell$ and $\lambda$ are continuous. Assume, furthermore, that the following conditions hold: 
(a) There exists a set $\mathbb{X}_{0} \subseteq \mathbb{X}$ of initial values such that

$$
\sup _{x \in \mathbb{X}_{0}, N \in \mathbb{N}} V_{N}(x)<\infty .
$$

(b) There are $\varepsilon>0$ and $\bar{N} \in \mathbb{N}$ such that Assumption 5.2 holds on the sets

$$
\mathbb{Z}_{N}:=\left\{(x, \bar{x}) \in B_{\varepsilon}\left(x^{e}\right) \times B_{\varepsilon}\left(x^{e}\right) \mid x_{u}(N, x)=\bar{x} \text { for some } u \in \mathcal{U}^{N}(x)\right\}
$$

with $\tilde{\gamma}_{N}, \tilde{\delta}_{N}$ decreasing in $N$.

Then Assumption 5.2 holds for $\bar{N}$ from (b) and $\mathbb{Z}_{N}$ from (5.8) with $\gamma_{N}, \delta_{N}$ decreasing in $N$.

Proof. Consider an initial value $x_{0} \in \mathbb{X}_{0}, N^{\prime} \geq \bar{N}$ and an optimal trajectory $x_{u_{x_{0}, N^{\prime}}^{\star}}\left(\cdot, x_{0}\right)$. We have to show $(5.4)$ for all $(x, \bar{x})$ of the form

$$
x=x_{u_{x_{0}, N^{\prime}}^{\star}}\left(k, x_{0}\right), \quad \bar{x}=x_{u_{x_{0}, N^{\prime}}^{\star}}\left(k+N, x_{0}\right)
$$

for $x_{0} \in \mathbb{X}_{0}, N=\bar{N}, \ldots, N^{\prime}$ and $k=0, \ldots, N^{\prime}-N$. The relation (5.2) implies the identity

$$
\widetilde{V}_{N^{\prime}}\left(x_{0}, x_{u_{x_{0}, N^{\prime}}^{\star}}\left(N^{\prime}, x_{0}\right)\right)=V_{N^{\prime}}\left(x_{0}\right)+\lambda\left(x_{0}\right)-\lambda\left(x_{u_{x_{0}, N^{\prime}}^{\star}}\left(N^{\prime}, x_{0}\right)\right)
$$

and by Condition (a), compactness of $\mathbb{X}$ and continuity of $\lambda$ the values $C_{V}:=$ $\sup _{x \in \mathbb{X}_{0}, N \in \mathbb{N}} V_{N}(x)$ and $C_{\ell}:=2 \max _{x \in \mathbb{X}}|\lambda(x)|$ are finite and we obtain

$$
\widetilde{V}_{N^{\prime}}\left(x_{0}, x_{u_{x_{0}, N^{\prime}}^{\star}}\left(N^{\prime}, x_{0}\right)\right) \leq C_{V}+C_{\ell} .
$$

Since $\tilde{\ell}(x, u) \geq 0$, for all $(x, \bar{x})$ of the form $(6.3)$ this implies

$$
\widetilde{V}_{N}(x, \bar{x}) \leq \widetilde{V}_{N^{\prime}}\left(x_{0}, x_{u_{x_{0}, N^{\prime}}^{\star}}\left(N^{\prime}, x_{0}\right)\right) \leq C_{V}+C_{\ell} .
$$

Now we define $\kappa:=\inf _{x \notin B_{\varepsilon}\left(x^{e}\right)} \tilde{\ell}^{\star}(x)>0$, fix $N$ and $k$ in (6.3) and consider three cases.

Case 1: $x \notin B_{\varepsilon}\left(x^{e}\right)$. In this case, we obtain

$$
\widetilde{V}_{N}(x, \bar{x}) \leq C_{V}+C_{\ell} \leq \frac{C_{V}+C_{\ell}}{\kappa} \tilde{\ell}^{\star}(x)
$$

which shows the assertion for $\gamma_{N}=\left(C_{V}+C_{\ell}\right) / \kappa$.

Case 2: $\bar{x} \notin B_{\varepsilon}\left(x^{e}\right)$. In this case, we obtain

$$
\widetilde{V}_{N}(x, \bar{x}) \leq C_{V}+C_{\ell} \leq \frac{C_{V}+C_{\ell}}{\kappa} \tilde{\ell}^{\star}(\bar{x})
$$

which shows the assertion for $\delta_{N}=\left(C_{V}+C_{\ell}\right) / \kappa+1$.

Case 3: $x, \bar{x} \in B_{\varepsilon}\left(x^{e}\right)$. In this case the desired inequality follows directly from Condition (b) with $\gamma_{N}=\tilde{\gamma}_{N}$ and $\delta_{N}=\tilde{\delta}_{N}$.

Hence, we obtain the assertion with $\gamma_{N}:=\max \left\{\left(C_{V}+C_{\ell}\right) / \kappa, \tilde{\gamma}_{N}\right\}$ and $\delta_{N}:=$ $\max \left\{\left(C_{V}+C_{\ell}\right) / \kappa+1, \tilde{\delta}_{N}\right\}$.

Now we can state the exponential turnpike theorem for nonlinear dissipative systems and compact $\mathbb{Y}$.

THEOREM 6.5. Consider a strictly dissipative optimal control problem (2.3), assuming without loss of generality $\ell\left(x^{e}, u^{e}\right)=0$ and $\lambda\left(x^{e}\right)=0$. Assume that $\mathbb{Y}$ is 
compact, that $f, \ell$ and $\lambda$ are continuous and that there are constants $C_{1}, C_{2}, p, \eta>0$ such that the inequalities

$$
C_{1}\left(|x|_{x^{e}}^{p}\right) \leq \tilde{\ell}(x, u) \leq C_{2}\left(|x|_{x^{e}}^{p}+|u|_{u^{e}}^{p}\right)
$$

hold for all $x \in B_{\eta}\left(x^{e}\right)$ and $u \in B_{\eta}\left(u^{e}\right)$. Assume, furthermore, that there exists $\varepsilon>0$ such that the following conditions hold.

(a) There exists a set $\mathbb{X}_{0} \subseteq \mathbb{X}$ and a $K \in \mathbb{N}$ such that for each $x \in \mathbb{X}_{0}$ there exists $k_{x} \leq K$ and a control $u_{x} \in \mathcal{U}^{k_{x}}(x)$ with $x_{u_{x}}\left(k_{x}, x\right) \in B_{\varepsilon}\left(x^{e}\right)$.

(b) There exists $\varepsilon>0$ and $N^{\prime} \in \mathbb{N}$ such that the system is controllable to and from $x^{e}$ on $B_{\varepsilon}\left(x^{e}\right)$ in $N^{\prime}$ steps in the following sense: there is $C>0$ such that for all $x \in B_{\varepsilon}\left(x^{e}\right)$ there exists $u_{1} \in \mathcal{U}^{N^{\prime}}(x)$ and $u_{2} \in \mathcal{U}^{N^{\prime}}\left(x^{e}\right)$ with

$$
x_{u_{1}}\left(N^{\prime}, x\right)=x^{e}, x_{u_{2}}\left(N^{\prime}, x^{e}\right)=x
$$

and

$$
\max \left\{\left|x_{u_{1}}(k)\right|_{x^{e}},\left|x_{u_{2}}(k)\right|_{x^{e}},\left|u_{1}(k)\right|_{u^{e}},\left|u_{2}(k)\right|_{u^{e}}\right\} \leq C|x|_{x^{e}}
$$

for $k=0, \ldots, N^{\prime}-1$.

Then the system has the exponential turnpike property on $\mathbb{X}_{0}$.

Proof. We show that the assumptions imply those of Theorem 5.6.

Condition (i) of Theorem 5.6 follows from the lower bound in (6.5), noting that for $|x|_{x^{e}} \geq \varepsilon$ the bound $\alpha$ in (4.1) can always be bounded from below by some polynomial since $\mathbb{Y}$ and thus $\mathbb{X}$ is compact.

In order to prove Condition (ii) of Theorem 5.6 we apply Proposition 6.4. To this end, we check that its assumptions hold.

Condition (a) of Proposition 6.4 follows by concatenating the control $u_{x}$ from (a) with the control $u_{1}$ from (b) for $x=x_{u_{x}}\left(k_{x}, x\right)$ and the constant control $u^{e}$. The resulting control $u$ satisfies $x_{u}\left(k_{x}+N^{\prime}, x\right)=x^{e}$ and thus

$$
\ell\left(x_{u}(k, x), u(k)\right) \leq \begin{cases}\max _{(x, u) \in \mathbb{Y}} \ell(x, u), & k=0, \ldots, k_{x}+N^{\prime}-1 \\ 0 & k \geq k_{x}+N^{\prime}\end{cases}
$$

which immediately implies

$$
V_{N}(x) \leq J_{N}(x, u) \leq\left(K+N^{\prime}\right) \max _{(x, u) \in \mathbb{Y}} \ell(x, u)
$$

for all $N \in \mathbb{N}$ and thus the desired uniform bound.

Condition (b) of Proposition 6.4 follows from Condition (b) of this theorem for $\bar{N}=2 N^{\prime}:$ For $x, \bar{x} \in B_{\varepsilon}\left(x^{e}\right)$ and $N \geq \bar{N}$ we define the control

$$
u(k):= \begin{cases}u_{1}(k), & k=0, \ldots, N^{\prime}-1 \\ u^{e}, & k=N^{\prime}, \ldots, N-N^{\prime}-1 \\ u_{2}\left(k+N-N^{\prime}\right), & k=N-N^{\prime}, \ldots, N-1\end{cases}
$$

with $u_{1}$ and $u_{2}$ from the assumption with $x$ for $u_{1}$ and $x=\bar{x}$ for $u_{2}$. Then the estimates from the assumption and the lower bound in (6.5) (reducing $\varepsilon$ if necessary to guarantee $C \varepsilon<\eta$ ) imply

$$
\tilde{\ell}\left(x_{u}(k, x), u(k)\right) \leq \begin{cases}C_{u}|x|_{x^{e}}^{p}, & k=0, \ldots, N^{\prime}-1 \\ 0, & k=N^{\prime}, \ldots, N-N^{\prime}-1 \\ C_{u}|\bar{x}|_{x^{e}}^{p}, & k=N-N^{\prime}, \ldots, N-1\end{cases}
$$


for some suitably chosen constant $C_{u}>0$ and thus $\widetilde{V}_{N}(x, \bar{x}) \leq N^{\prime} C_{u}\left(|x|_{x^{e}}^{p}+|\bar{x}|_{x^{e}}^{p}\right)$ which, using lower bound in (6.5), is less or equal $N^{\prime} C_{u} / C_{1}\left(\tilde{\ell}^{\star}(x)+\tilde{\ell}^{\star}(\bar{x})\right)$ and thus shows Condition (b) of Proposition 6.4 with $\gamma_{N}=N^{\prime} C_{u} / C_{1}$ and $\delta_{N}=N^{\prime} C_{u} / C_{1}+1$. Thus, Proposition 6.4 applies and implies Condition (ii) of Theorem 5.6.

Finally, Condition (iii-a) of Theorem 5.6 follows since $\tilde{\ell}$ is continuous and $\mathbb{Y}$ is compact, hence $\tilde{\ell}$ is bounded on $\mathbb{Y}$ and thus $\tilde{\ell}^{\star}$ is bounded on $\mathbb{X}$.

Hence, all assumptions of Theorem 5.6 hold and we can conclude the desired exponential turnpike property.

Theorem 6.5 can be used in order to prove the exponential turnpike property for Example 2.3: The problem is strictly dissipative, all functions are continuous and (6.5) follows since $\tilde{\ell}$ is a polynomial. Condition (a) holds on $\mathbb{X}_{0}=\mathbb{X}$ for $K=1$ by setting $u_{x}(0)=-2 x$. Condition (b) holds with $N^{\prime}=1$ by setting $u_{1}(0)=-2 x$ and $u_{2}(0)=x$.

7. Conclusion. We have derived a condition for establishing an exponential turnpike property for nonlinear discrete time finite horizon optimal control problems, given in terms of a bound on an auxiliary optimal value function. The condition applies to strictly dissipative systems, a property which we have shown to hold for linear quadratic systems under mild regularity conditions without having to assume strict convexity of the stage cost of the optimal control problem. For unconstrained affine linear quadratic problems with strictly convex stage cost our condition is equivalent to stabilizability of the underlying control systems and for general nonlinear problems with compact constraints it can be concluded from suitable controllability properties.

8. Appendix: A result on positive definite matrices. In this section we provide an auxiliary result needed in the proof of Lemma 4.4. We first consider the discrete-time Lyapunov equation $P-A^{T} P A=Y$ with $Y>0$. Note that this equation has a unique positive definite solution, if $\sigma(A) \subset \mathbb{C}_{<1}$ and a unique negative definite solution, if $\sigma(A) \subset \mathbb{C}_{>1}$. More generally, we obtain the following discrete time version of a continuous time result in [17, Theorem 2.4.10]. The proof is provided for the sake of completeness.

Lemma 8.1. For $A \in \mathbb{R}^{n \times n}$ the following are equivalent:

(a) $\sigma(A) \cap \mathbb{C}_{=1}=\emptyset$

(b) $\exists Y=Y^{T}>0, P=P^{T}: P-A^{T} P A=Y$

Proof. '(b) $\Rightarrow(\mathrm{a})^{\prime}$ : Assume that (b) holds, but not (a), i.e. $A x=\lambda x$ for some $x \in \mathbb{C}^{n},|\lambda|=1$. Then

$$
0<x^{*} Y x=x^{*}\left(P-A^{T} P A\right) x=\left(1-|\lambda|^{2}\right) x^{*} P x=0,
$$

which is a contradiction.

'(a) $\Rightarrow$ (b)': If $\sigma(A) \subset \mathbb{C}_{<1} \cup \mathbb{C}_{>1}$, then there exists a similarity transformation with some nonsingular $S$, such that

$$
\tilde{A}=S^{-1} A S=\left[\begin{array}{ll}
A_{+} & \\
& A_{-}
\end{array}\right], \quad \text { where } \quad \sigma\left(A_{+}\right) \subset \mathbb{C}_{>1}, \sigma\left(A_{-}\right) \subset \mathbb{C}_{<1} .
$$

Substituting $\tilde{P}=S^{T} P S$ for $P$ and $\tilde{Y}=S^{*} Y S$ for $Y$ we may assume that $A=\tilde{A}$. For any block-diagonal positive definite $\tilde{Y}=\operatorname{diag}\left(Y_{1}, Y_{2}\right)$, we find a suitable Hermitian $\tilde{P}=\operatorname{diag}\left(P_{1}, P_{2}\right)$ with $P_{1}-A_{+}^{T} P_{1} A_{+}=Y_{1}, P_{2}-A_{+}^{T} P_{2} A_{+}=Y_{2}$, where $P_{1}<0$ and $P_{2}>0$. प

Now let also $Q$ and $B$ be given and consider the map

$$
(x, u) \mapsto u^{T} Q u+x^{T} P x-\left(x^{T} A^{T}+u^{T} B^{T}\right) P(A x+B u) .
$$


The following proposition states the result needed in the proof of Lemma 4.4.

Proposition 8.2. If $\sigma(A) \cap \mathbb{C}_{=1}=\emptyset$ and $Q>0$ then there exists a symmetric matrix $P$ such that (8.1) is strictly convex.

Proof. By Lemma 8.1 there exists a matrix $\widetilde{P}$ satisfying $\widetilde{P}-A^{T} \widetilde{P} A>0$. Then, obviously, $\alpha \widetilde{P}-A^{T} \alpha \widetilde{P} A>0$ for each $\alpha>0$. Setting $P:=\alpha \widetilde{P}$, the expression in (8.1) becomes

$$
\begin{aligned}
& u^{T} Q u+x^{T} P x-\left(x^{T} A^{T}+u^{T} B^{T}\right) P(A x+B u) \\
& =\left[\begin{array}{l}
x \\
u
\end{array}\right]^{T}\left[\begin{array}{cc}
\alpha\left(\widetilde{P}-A^{T} \widetilde{P} A\right) & -\alpha A^{T} \widetilde{P} B \\
-\alpha B^{T} \widetilde{P} A & Q-\alpha B^{T} \widetilde{P} B
\end{array}\right]\left[\begin{array}{l}
x \\
u
\end{array}\right] .
\end{aligned}
$$

Hence, (8.1) is strictly convex if the matrix in (8.2) is positive definite, which in turn holds if its Schur-complement is positive definite, i.e. if

$$
\begin{aligned}
0 & <Q-\alpha B^{T} \widetilde{P} B-\alpha B^{T} \widetilde{P} A\left(\alpha\left(\widetilde{P}-A^{T} \widetilde{P} A\right)\right)^{-1} \alpha A^{T} \widetilde{P} B \\
& =Q-\alpha\left(B^{T} \widetilde{P} B+B^{T} \widetilde{P} A\left(\widetilde{P}-A^{T} \widetilde{P} A\right)^{-1} A^{T} \widetilde{P} B\right) .
\end{aligned}
$$

Since $Q>0$, this holds if we choose $\alpha>0$ sufficiently small.

\section{REFERENCES}

[1] R. Amir and I. Evstigneev, Stochastic version of Polterovich's model: exponential turnpike theorems for equilibrium paths, Macroecon. Dyn., 3 (1999), pp. 149-166.

[2] R. Amrit, J. B. Rawlings, and D. Angel, Economic optimization using model predictive control with a terminal cost, Annual Rev. Control, 35 (2011), pp. 178-186.

[3] B. D. O. Anderson And P. V. Kокотоvić, Optimal control problems over large time intervals, Automatica, 23 (1987), pp. 355-363.

[4] D. Angeli, R. Amrit, AND J. B. Rawlings, Receding horizon cost optimization for overly constrained nonlinear plants, in Proceedings of the 48th IEEE Conference on Decision and Control - CDC 2009, Shanghai, China, 2009, pp. 7972-7977.

[5] D. Angeli and J. B. Rawlings, Receding horizon cost optimization and control for nonlinear plants, in Proceedings of the 8th IFAC Symposium on Nonlinear Control Systems NOLCOS 2010, Bologna, Italy, 2010, pp. 1217-1223.

[6] T. Bewley, An integration of equilibrium theory and turnpike theory, J. Math. Econom., 10 (1982), pp. 233-267.

[7] S. P. Boyd and L. Vandenberghe, Convex Optimization, Cambridge University Press, 2004.

[8] D. A. CARLson, The existence of catching-up optimal solutions for a class of infinite horizon optimal control problems with time delay, SIAM J. Control Optim., 28 (1990), pp. 402-422.

[9] D. A. Carlson, A. B. Haurie, and A. Leizarowitz, Infinite horizon optimal control Deterministic and Stochastic Systems, Springer-Verlag, Berlin, second ed., 1991.

[10] M. Diehl, R. Amrit, and J. B. Rawlings, A Lyapunov function for economic optimizing model predictive control, IEEE Trans. Autom. Control, 56 (2011), pp. 703-707.

[11] R. Dorfman, P. A. Samuelson, And R. M. Solow, Linear Programming and Economic Analysis, Dover Publications, New York, 1987. Reprint of the 1958 original.

[12] L. GRüNE, Analysis and design of unconstrained nonlinear MPC schemes for finite and infinite dimensional systems, SIAM J. Control Optim., 48 (2009), pp. 1206-1228.

[13] - Economic receding horizon control without terminal constraints, Automatica, 49 (2013), pp. $725-734$.

[14] L. Grüne and J. Pannek, Nonlinear Model Predictive Control. Theory and Algorithms, Springer-Verlag, London, 2011.

[15] L. Grüne, J. Pannek, M. Seehafer, and K. Worthmann, Analysis of unconstrained nonlinear MPC schemes with varying control horizon, SIAM J. Control Optim., 48 (2010), pp. 4938-4962.

[16] L. GRÜne And A. Rantzer, On the infinite horizon performance of receding horizon controllers, IEEE Trans. Automat. Control, 53 (2008), pp. 2100-2111. 
[17] R. A. Horn And C. R. Johnson, Topics in matrix analysis, Cambridge University Press, Cambridge, 1994. Corrected reprint of the 1991 original.

[18] H. K. Khalil, Nonlinear Systems, Prentice Hall, 3rd ed., 2002.

[19] M. A. Mamedov, A turnpike theorem for continuous-time control systems when the optimal stationary point is not unique, Abstr. Appl. Anal., (2003), pp. 631-650.

[20] R. Marimon, Stochastic turnpike property and stationary equilibrium, Journal of Economic Theory, 47 (1989), pp. 282-306.

[21] L. W. McKenZIE, Optimal economic growth, turnpike theorems and comparative dynamics, in Handbook of Mathematical Economics, Vol. III, vol. 1 of Handbooks in Econom., NorthHolland, Amsterdam, 1986, pp. 1281-1355.

[22] J. von Neumann, A model of general economic equilibrium, The Review of Economic Studies, 13 (1945), pp. 1-9.

[23] A. Porretta And E. Zuazua, Long time versus steady state optimal control, SIAM J. Control Optim., 51 (2013), pp. 4242-4273. To appear.

[24] R.T. Rockafellar, Convex Analysis, vol. 28 of Princeton Mathematical Series, Princeton University Press, Princeton, NJ, 2nd ed., 1972.

[25] E. D. Sontag, Mathematical Control Theory, Springer Verlag, New York, 2nd ed., 1998.

[26] J. C. Willems, Dissipative dynamical systems. I. General theory, Arch. Rational Mech. Anal., 45 (1972), pp. 321-351.

[27] - Dissipative dynamical systems. II. Linear systems with quadratic supply rates, Arch. Rational Mech. Anal., 45 (1972), pp. 352-393.

[28] A.J. Zaslavski, Turnpike Properties in the Calculus of Variations and Optimal Control, Springer, New York, 2006. 